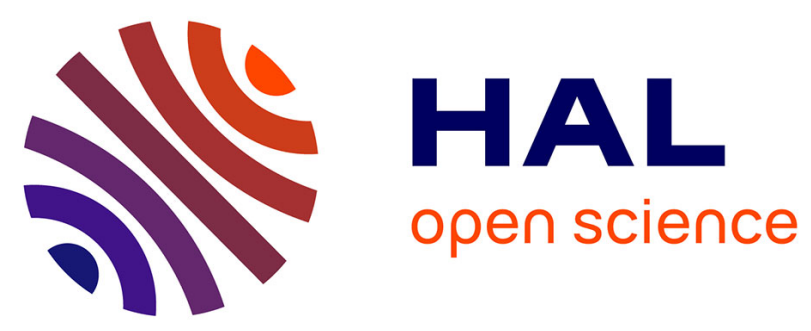

\title{
Les céréales à paille, hôtes de Meloidogyne naasi Franklin. I. - Mise au point de méthodes et résultats préliminaires d'évaluation de la résistance ou du caractère multiplicateur
}

Françoise Person-Dedryver, Danielle Pannetier

\section{To cite this version:}

Françoise Person-Dedryver, Danielle Pannetier. Les céréales à paille, hôtes de Meloidogyne naasi Franklin. I. - Mise au point de méthodes et résultats préliminaires d'évaluation de la résistance ou du caractère multiplicateur. Agronomie, 1984, 4 (10), pp.977-985. hal-00884601

\author{
HAL Id: hal-00884601 \\ https://hal.science/hal-00884601
}

Submitted on 1 Jan 1984

HAL is a multi-disciplinary open access archive for the deposit and dissemination of scientific research documents, whether they are published or not. The documents may come from teaching and research institutions in France or abroad, or from public or private research centers.
L'archive ouverte pluridisciplinaire HAL, est destinée au dépôt et à la diffusion de documents scientifiques de niveau recherche, publiés ou non, émanant des établissements d'enseignement et de recherche français ou étrangers, des laboratoires publics ou privés. 


\title{
Les céréales à paille, hôtes de Meloidogyne naasi Franklin. I. - Mise au point de méthodes et résultats préliminaires d'évaluation de la résis- tance ou du caractère multiplicateur
}

\author{
Françoise PERSON-DEDRYVER \\ avec la collaboration technique de Danielle PANNETIER \\ I.N.R.A., Laboratoire de Zoologie, Centre de Recherches de Rennes, B.P. 29, F 35650 Le Rheu
}

L'évaluation du caractère multiplicateur ou résistant de différentes céréales vis-à-vis de Meloidogyne naasi nécessite la mise au point de méthodes de jugement adaptées aux conditions de culture. Les tests proposés sont réalisés, soit sur sol conservé à l'extérieur, soit en conditions entièrement contrôlées, dans des boîtes de Petri. L'analyse critique des formations de femelles, œufs et galles du nématode, en fonction du potentiel infectieux et de la variété, démontre la stabilité des tests préconisés indépendamment de leurs conditions de réalisation. Elle permet la mise au point d'un mode d'emploi des 2 méthodes.

En sol infesté, les tests donnent les taux de multiplication de $M$. naasi sur diverses céréales, 4 à 9 mois après le semis des caryopses étudiés. Pour réaliser un bon test, il est préférable de faire 4 répétitions par variété, de prendre 2 témoins de multiplication dans les orges ou le blé et 1 de résistance choisi parmi les avoines. Les dénombrements de femelles et œufs du nématode en juillet sont les meilleurs critères d'appréciation du caractère hôte des céréales à paille. La distinction entre 2 niveaux de multiplication se fait plus aisément au plus fort potentiel infectieux éprouvé, voisin de 18 à $23 \mathrm{~L} 2$.

Les tests réalisés en boîtes de Petri, faciles à analyser en 1,5 mois, semblent plus particulièrement adaptés à l'analyse d'un grand nombre de variétés. L'inoculation à l'aide d'une seringue, des plantes à tester par une cinquantaine de larves infectieuses et le stockage des boîtes 1,5 mois à $23{ }^{\circ} \mathrm{C}$, peuvent être employés pour la recherche de géniteurs de résistance, la détection de lignées résistantes par cette méthode étant aisée. La $2^{\mathrm{e}}$ technique, consistant à déposer une à une $3 \times 16$ larves par plantule sur la gélose et à stocker les boîtes à $23{ }^{\circ} \mathrm{C}$, est plus adaptée aux études du mode d'hérédité de la résistance, le caractère hôte de chaque plante testée étant systématiquement détecté.

Les résultats préliminaires obtenus par ces tests sur le comportement de 2 orges, 1 blé et 1 avoine d'hiver, de 2 orges de printemps et d'l lignée d'Aegilops ventricosa Tausch. sont analysés et comparés entre eux.

Mots clés additionnels : Nématode, orge, blé, avoine, Aegilops ventricosa. susceptibility and preliminary results.

Test methods to evaluate the host efficiency of different cereals for Meloidogyne naasi are necessary to detect varieties which are resistant to this nematode in our climatic and agricultural conditions. These new techniques involve rearing cereals either in infested soil or under controlled conditions in Petri dishes.

Consistent results were obtained on the development of the females, eggs and galls of the nematode according to inoculum level and variety. The methods could therefore be developed for practical use.

In infested soil, the degree of susceptibility of different cereal varieties was estimated 4 or 9 months after the sowing of tested plants. For successful use of the test, 4 replicates were needed per species or variety. Two susceptible controls were selected from the good hosts of the nematode in barley or wheat and one resistant control from oat. Female and egg numbers in July gave the best estimation of the host reaction of cereals to $M$. naasi. The distinction between 2 levels of host reaction was clearest at the highest infestation level (18-23 second stage larvae per $g$ of soil).

The tests under controlled conditions in Petri dishes with plants grown on agar were reliable, easy to conduct and gave a quick answer, 6 weeks after the sowing of test plants. In one technique, the plants were individually infested with $3 \times 16$ second stage larvae which were placed just behind the root tip and the Petri dishes held for 6 weeks in a growth chamber at $23{ }^{\circ} \mathrm{C}$. This first technique would be most suitable for studying the mode of inheritance of resistance, since in this case the host reaction of each individual tested plant is found. The second method consisted of an injection with a hypodermic syringe of some 50 second stage larvae onto the agar of each Petri dish then incubated as above. This second technique would be useful in detecting lines or varieties resistant to $M$. naasi.

Additional key words : Nematode, barley, wheat, oat, Aegilops ventricosa. 


\section{INTRODUCTION}

Meloidogyne naasi Franklin, nématode à galles des graminées, est responsable de chutes de rendement importantes sur orge aux Etats-Unis (ALLEN et al., 1970), en France (CAUBEL et al., 1972), en Belgique (GOORIS \& D'HERDE, 1977) et en Grande-Bretagne (YORK, 1980). Le blé n'est pas épargné par ce nématode et subit des dommages, plus particulièrement en Europe (CAUBEL et al., 1972 ; GOORIS \& D'HERDE, 1977) et en Amérique du Sud (KILPATRICK et al., 1976).

Les méthodes de lutte sont basées essentiellement sur l'utilisation de rotations de cultures incluant des plantes mauvais hôtes pour ce parasite, telles que l'avoine, la pomme de terre (GoORIS \& D'HERDE, 1977), l'emploi de nématicides restant trop onéreux en culture céréalière. Cependant, la rotation de cultures, faisant appel à des espèces assainissantes, n'est pas souvent économiquement rentable et reste peu utilisée dans les grandes zones céréalières. Il s'avère intéressant de rechercher des variétés de blé ou d'orge résistantes, qui, en s'opposant au développement des femelles du nématode, contribueraient au maintien des densités de populations infestantes en dessous du seuil de nuisibilité. Actuellement aucun cultivar français ou étranger, de blé ou d'orge, n'est connu pour être résistant au développement de $M$. naasi.

La mise au point de méthodes pour le jugement de la résistance ou du caractère multiplicateur des céréales vis-à-vis de ce parasite est indispensable pour pouvoir envisager des programmes de sélection de variétés résistantes. En France, des techniques d'évaluation de la résistance ont été élaborées pour d'autres nématodes nuisibles aux cultures céréalières, tels qu'Heterodera avenae Woll., Ditylenchus dipsaci Kühn et Pratylenchus spp. (RIVOAL et al., 1978). Elles n'existent pas vis-à-vis de $M$. naasi, alors qu'à l'étranger elles sont fréquemment citées dans les nombreux travaux consacrés aux plantes-hôtes de cette espèce.

La plupart des techniques utilisées sont cependant fastidieuses, car elles se basent sur l'infestation artificielle de sols préalablement stérilisés, soit par des quantités connues de larves infectieuses, soit par des galles induites par le nématode dont l'extraction exige un travail important (GoORIS \& D'HERDE, 1977; EDIZ \& DiCKERSON, 1976 ; Allen et al., 1970 ; SiDDIQUI \& TAYLOR, 1970 ; RADEWALD et al., 1970 ; MICHELl et al., 1973 ; ROBERTS et al., 1982).

Les études du comportement des céréales vis-à-vis de $M$. naasi, à l'exception de celle signalée par GooRIS \& D'HERDE (1977), sont toujours réalisées en serre entre 20 et $29^{\circ} \mathrm{C}$ (ALLEN et al., 1970 ; RADEWALD et al., 1970 ; SIDDIQUI \& TAYLOR, 1970 ; COOK, 1972 ; MICHELl et al., 1973 ; EDIZ \& DICKERSON, 1976 ; OGUNFOWORA, 1977 ; ROBERTS et al., 1982). Or, GOORIS \& D'HERDE (1977) ont montré que ces températures, excessives pour la culture de céréales, sont responsables des résultats contradictoires observés dans l'étude du caractère hôte de certaines céréales et notamment de l'avoine cultivée. Ainsi, Avena sativa $\mathrm{L}$. ne multiplie pas le nématode à $15^{\circ} \mathrm{C}$ ou en conditions de plein champ, alors qu'elle se comporte en hôte efficace en serre. Les conditions de culture de plein champ, employées par GoORIS \&
D'HERDE (1977), avec un semis réalisé début avril, correspondent à celles de nos variétés de printemps, mais diffèrent beaucoup de celles de nos variétés d'hiver.

Le travail entrepris concerne la mise au point de nos propres méthodes de jugement de la résistance ou $\mathrm{du}$ caractère multiplicateur des espèces et variétés de céréales les plus susceptibles d'être attaquées par le nématode en France (blés d'hiver et orges de printemps ou hiver). Les formations de femelles, d'œufs, de galles de $M$. naasi sont étudiées en fonction, principalement, du potentiel infectieux et de la variété. Les tests sont réalisés, soit sur sol infesté, soit en conditions entièrement contrôlées de boîtes de Petri. Ces derniers s'inspireront de ceux de PERSON \& DOUSSINAULT (1978) sur $H$. avenae et de SIDDIQUI \& TAYLOR (1970), qui ont étudié la formation des galles induites par $M$. naasi sur 1 variété d'avoine cultivée en boîtes de Petri. Ils doivent, dans les 2 cas, être faciles à mettre en place et à analyser et donner des résultats fiables correspondant à nos conditions de culture de plein champ.

\section{MATÉRIEL ET MÉTHODES}

\section{A. Matériel}

\section{Le nématode}

Le sol naturellement infesté en $M$. naasi, prélevé au fur et à mesure des besoins, provient de monocultures de blés d'hiver, "Roazon " ou "Champlein », dans des essais de longue durée de la Station expérimentale du Rheu (Ille-et-Vilaine).

Les larves infectieuses L2 fraîchement écloses sont issues de galles recueillies, en septembre après culture, sur les racines des blés " Roazon » et " Champlein ». Les galles sont conservées au froid $\left(5\right.$ à $\left.7{ }^{\circ} \mathrm{C}\right)$ puis dilacérées et traitées $25 \mathrm{mn}$ à l'hypochlorite de sodium 4 p. 1000 , puis $25 \mathrm{mn}$ à l'acide ascorbique 4 p. 1000 , afin de lever la diapause des larves L2 (OGUNFOWORA \& EvANS, 1977). Leur éclosion massive est obtenue à la température du laboratoire, dans de l'eau distillée, dès le $2^{\mathrm{e}}$ jour.

\section{Les plantes}

Les cultivars d'orge, de blé et d'avoine choisis sont inscrits au catalogue français des variétés au cours de la période 1978-1983, à l'exception de l'avoine suédoise "Nelson " présentant une résistance à $H$. avenae.

Pour chaque céréale bon hôte de $M$. naasi (orge et blé), nous avons retenu des variétés qui le multiplient peu, moyennement ou fortement. Dans nos tests, nous avons ainsi la possibilité de juger différents niveaux de taux de multiplication du nématode.

Les variétés d'avoine sont prises dans chaque test comme témoin non multiplicateur puisqu'elles sont considérées résistantes en Europe, par FRANKLIN (1965), CAUBel et al. (1972) et GoORIS \& D'HERDE (1977).

Une espèce sauvage, voisine du blé, a été introduite dans nos essais : il s'agit d'Aegilops ventricosa Tausch. $\mathrm{n}^{\circ} 11$ présentant une résistance à $H$. avenae (DOSBA \& RIVOAL, 1981). 


\section{B. Méthodes}

\section{Tests réalisés sur sol naturellement infesté}

Pour une même variété, plusieurs tests sont réalisés, soit en pots, soit directement au champ, indépendamment de l'espèce végétale étudiée et de l'année de culture. Ils sont au nombre de 4 pour les variétés d'orge de printemps et de 2 pour les variétés de blé et d'orge d'hiver.

\section{a) Mise en place des tests}

Des bouteilles en matière plastique d'1,5 1, renversées, dont le fond a été préalablement découpé, sont remplies avec 11 de sol puis placées en conditions extérieures dès début septembre. Les caryopses, prégermés, sont semés fin octobre ou début novembre pour les variétés de type hiver et en mars pour celles de type printemps. $A$. ventricosa est semé indifféremment en octobre ou en mars. Quatre répétitions sont effectuées par variété à tester. En cours de culture, les plantes reçoivent une fois par mois un engrais complet sous forme liquide.

Les essais au champ sont réalisés exclusivement en 1980-1981, en dispositifs factoriels à 4 blocs avec des parcelles de $1,3 \times 4,5 \mathrm{~m}$. Les semis sont réalisés le 20 novembre pour les blés d'hiver et le 6 avril pour les orges de printemps. Les cultures sont conduites selon la pratique courante locale avec une fertilisation azotée de 90 unités/ha.

\section{b) Estimation du potentiel infectieux avant culture}

Des échantillons de $200 \mathrm{~g}$ de sol correspondant soit à 5 prélèvements pris au hasard à l'aide d'un déplantoir dans chaque parcelle de l'essai bloc, soit à $20 \mathrm{~kg}$ de sol servant à remplir des bouteilles, sont stockés au froid $\left(7^{\circ} \mathrm{C}\right)$ durant 2 mois, puis entreposés durant $10 \mathrm{j}$ à $20^{\circ} \mathrm{C}$ pour obtenir l'éclosion massive des larves L2 (OGUNFOWORA \& EVANS, 1977).

L'extraction des larves L2 du sol s'effectue par la méthode centrifugation-flottaison (COOLEN \& D'HERDE, 1972) sur la moitié du sol prélevé, l'autre servant à déterminer le poids sec. Les larves L2 sont dénombrées au microscope stéréoscopique et leur nombre exprimé par gramme de sol sec.

Deux niveaux de potentiel infectieux ont été choisis : l'un faible et proche du seuil de nuisibilité, voisin de 3 à $7 \mathrm{~L} 2 / \mathrm{g}$ de sol (PERSON-DEDRYVER, 1983), l'autre fort et situé entre 18 et $23 \mathrm{~L} 2 / \mathrm{g}$ de sol.

\section{c) Notations}

Les notations sont effectuées uniquement en juillet pour toutes les variétés cultivées au champ, ainsi que pour les orges et blés de type hiver semés dans les bouteilles. L'analyse est réalisée en juillet ou en septembre pour $A$. ventricosa 11 et les variétés d'orge de printemps, dans l'espoir de proposer 2 dates possibles d'examen des populations de nématodes endoracinaires. Les plantes prélevées dans les bouteilles ou au champ à la bêche, à raison d'une dizaine prises au hasard par parcelle, sont lavées ; différentes notations sont alors réalisées.

Lors de la $1^{\text {re }}$ année d'étude (1978-1980), on a séparé les systèmes racinaires selon leur poids supé- rieur ou inférieur à $5 \mathrm{~g}$. Les plantes appartenant à cette $2^{\mathrm{e}}$ catégorie sont toutes pesées. A partir de 1980 , le poids des systèmes racinaires est systématiquement enregistré.

Les indices de galles sont notés pour chaque plante et correspondent à l'échelle suivante :

$\begin{array}{cc}\text { indice (i) } & \text { nombre de galles par plante } \\ 0 & 0 \\ 1 & 1 \text { à } 10 \\ 2 & 11 \text { à } 25 \\ 3 & 26 \text { à } 50 \\ 4 & 51 \text { à } 100 \\ 5 & \text { supérieur à } 100\end{array}$

Un indice pondéré d'abondance de galles est calculé pour chaque répétition du test et chaque variété. Cet indice est défini en fonction du nombre variable $\mathrm{N}$ de plantes sur lequel ont porté les notations, des fréquences $\left(f_{i}\right)$ relatives aux indices (i) affectés à chaque plante et du nombre de classes d'indice $i, C=6$ (CAUBEL et al., 1971).

Indice pondéré d'abondance de galles

$$
=\frac{\sum \mathrm{i} \times \mathrm{f}_{\mathrm{i}} \times 100}{\mathrm{~N}(\mathrm{C}-1)}=\frac{\sum \mathrm{i} \times \mathrm{f}_{\mathrm{i}} \times 100}{\mathrm{~N} \times 5} .
$$

L'extraction des femelles et des œufs de $M$. naasi est réalisée sur un poids racinaire le plus proche possible de $5 \mathrm{~g}$, correspondant, soit à un, soit à plusieurs systèmes racinaires. Les $5 \mathrm{~g}$ de racines à analyser sont coupés en petits morceaux de 1 à $2 \mathrm{~cm}$ de long et broyés durant 2 fois $30 \mathrm{~s}$ à la vitesse lente $(15000 \mathrm{t} / \mathrm{mn})$ d'un mixeur WARING. Les femelles et les œufs en sont extraits par la méthode de centrifugation-flottaison. Ils sont ensuite comptés au microscope stéréoscopique, soit en totalité, soit sur une partie aliquote quand ils sont en grand nombre. Pour faciliter les comparaisons entre essais, le nombre de femelles est exprimé par $\mathrm{g}$ de racine alors que nous rapportons le nombre d'œufs à celui des femelles.

\section{d) Analyse statistique des résultats}

Des analyses de variance ont été faites pour déterminer l'influence des différents facteurs testés : date d'extraction, potentiel infectieux, variétés. Les données sont transformées en $\log x$ ou $\log x+1$, lorsque les variances ne sont pas homogènes. Par nécessité, il a été procédé à une subdivision des effets de chaque facteur, en fonction du nombre de degrés de liberté, par la méthode des contrastes (DAGNELIE, 1970).

\section{Tests réalisés en boîtes de Petri}

Les caryopses de céréales, préalablement désinfectés dans une solution alcoolique de chlorure mercurique à 2 p. 100 , sont cultivés sur milieu eau agar à 2 p. 100 , coulé en boîtes de Petri selon la méthode précédemment décrite pour $H$. avenae (PERSON \& DoussiNAULT, 1978).

Deux techniques sont utilisées pour déposer les larves infestantes dans les boîtes de Petri, lorsque les 3 premières racines ont 1,5 à $3 \mathrm{~cm}$ de long. Dans la $1^{\text {re }}$ technique, on dépose les larves L2 une à une sur la 
gélose au niveau de la coiffe des 3 racines. 3 quantités d'inoculum sont choisies : 8, 12 ou $16 \mathrm{~L} 2$ par racine, soit 24,36 et $48 \mathrm{~L} 2$ par plante. La $2^{\text {e }}$ technique consiste à verser $1 \mathrm{ml}$ d'une suspension aqueuse contenant une cinquantaine de larves $\mathrm{L} 2$, directement sur la gélose à l'aide d'une seringue. Cette suspension est ensuite étalée sur une grande partie de la surface du milieu gélosé par inclinaison de la boîte. Dix répétitions sont faites par variété.

Toutes les boîtes sont placées à $20^{\circ} \mathrm{C}$ durant $24 \mathrm{~h}$ après le dépôt des larves afin d'obtenir une pénétration identique. Après ce temps, les boîtes sont réparties dans 2 enceintes climatisées à 20 ou $23^{\circ} \mathrm{C}$ et toutes 2 avec $16 \mathrm{~h}$ d'éclairement par $24 \mathrm{~h}$.

Après un délai d'un mois et demi, qui est nécessaire au développement complet des femelles, les boîtes sont examinées. Chaque galle est extraite des boîtes de Petri et disséquée afin de dénombrer les femelles et les masses d'œufs formées sur les plantes. Cette analyse des plantes cultivées en boîtes de Petri est plus rapide que celle des plantes semées sur sol infesté.

\section{RÉSULTATS}

\section{A. Tests réalisés sur sol naturellement infesté}

\section{Qualité d'hôte des variétés d'orge et d'avoine de printemps}

L'avoine «Noire de Moyencourt » présente un grand nombre de plantes dépourvues de femelles (tabl. 1). Un faible nombre de femelles est parfois observé sur quelques plantes, mais très peu d'œufs sont dénombrés : 23 par femelle au maximum. Les indices pondérés de galles de ce cultivar sont inférieurs à ceux des orges en 1979 et 1980, mais les avoisinent en 1981.

TABLEAU 1

Influence des facteurs date d'extraction, potentiel infectieux et variété d'orge et d'avoine de printemps sur la formation des femelles, des aufs et des galles de Meloidogyne naasi et sur le poids des racines de chaque plante.

Influence of extraction date, degree of infection and spring variety of barley and oat on the production of females, eggs and galls of Meloidogyne naasi and on the root weight of each plant.

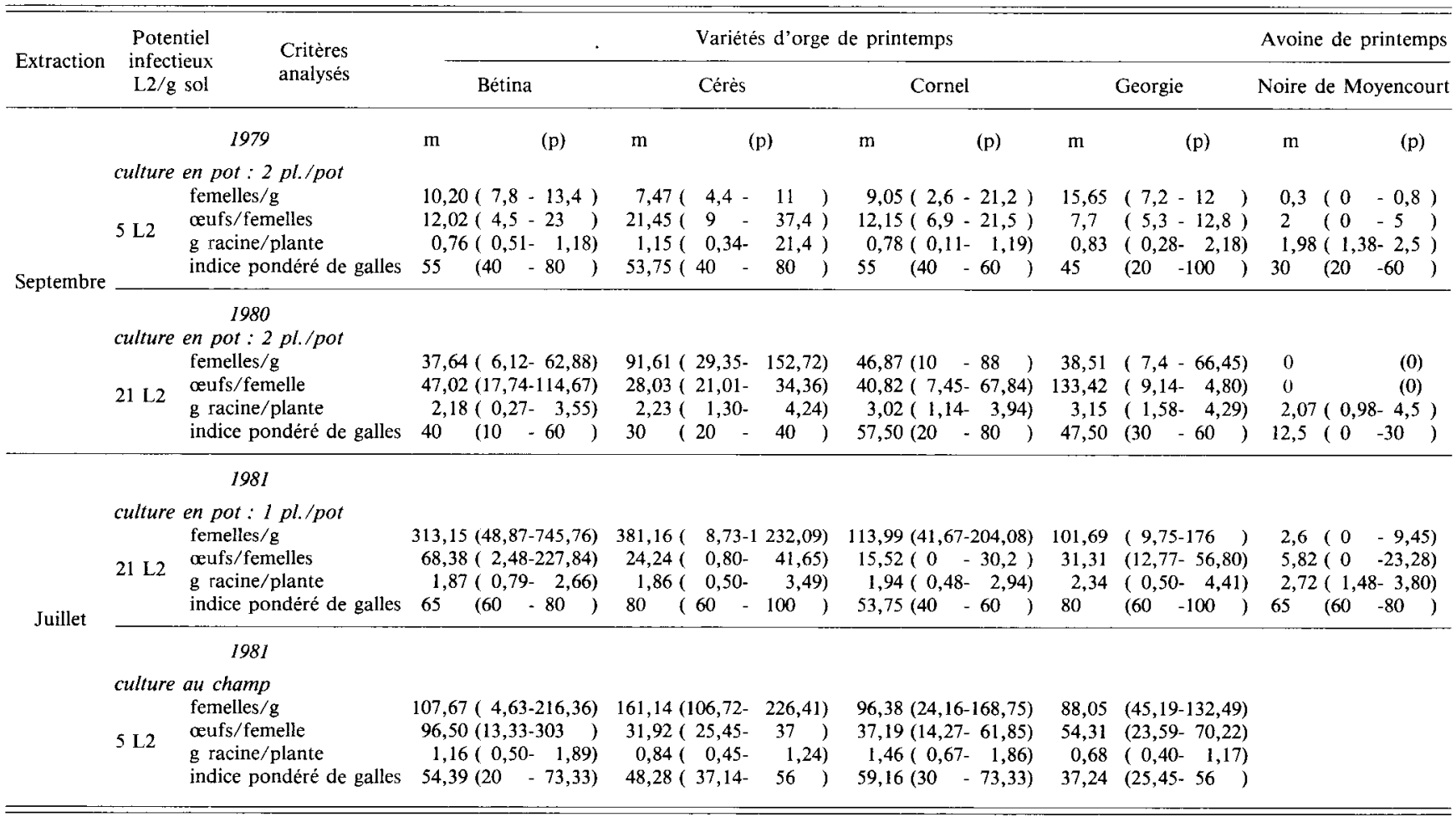

$m=$ moyenne sur 4 répétitions

$\mathrm{p}=$ valeurs extrêmes : minimale et maximale

Les résultats obtenus avec les orges sont plus complexes et les formations de femelles, d'œufs et de galles du nématode seront analysées successivement (tabl. 1).

Le nombre de femelles comptées par g de racine est significativement plus important $(F=41,8)$ lorsque les extractions sont réalisées en juillet. Pour un même mois d'extraction, il y a significativement plus de femelles formées à fort qu'à faible potentiel infectieux $(F=11,6)$. L'analyse statistique ne met pas en évidence d'effets significatifs du facteur variétal. Cepen- dant, pour les expériences avec extraction réalisées en juillet, nous conservons le même ordre croissant dans les nombres de femelles formées sur les variétés classées comme suit : « Georgie », « Cornel », « Bétina ", "Cérès », cette dernière étant la variété qui produit le plus de femelles, quelle que soit l'expérience.

Les facteurs potentiels infectieux, date d'extraction, variétés, n'ont pas d'effet significatif sur le nombre d'œufs formés par femelle.

Les indices pondérés de galles enregistrés sur les plantes en juillet sont significativement supérieurs à 
ceux notés en septembre $(F=6,5)$. Le potentiel infectieux et les variétés n'induisent pas de différence significative dans la formation des galles.

Les poids racinaires des orges, enregistrés par plante, sont différents d'une expérience à une autre (tabl. 1) :

- les poids racinaires des plantes de l'essai champ 1981 sont significativement plus faibles que ceux des plantes des essais pots 1979,1980 et $1981(F=8)$;

- il n'y a pas de différence significative entre les poids racinaires par plante enregistrés, d'une part, en septembre dans les essais 1979 et 1980 à 2 plantes par pot, d'autre part, en juillet dans l'expérience 1981 à 1 plante par pot ;

- les poids racinaires des plantes examinées en septembre des 2 essais pot 1979 et 1980 diffèrent significativement entre eux $(F=25,4)$.

\section{Qualité d'hôte des variétés d'hiver}

\section{a) Les orges et avoines}

Les plantes de la variété d'avoine «Hiver du Prieuré » sont pour la plupart totalement résistantes à M. naasi (tabl. 2) ; seules quelques femelles peuvent se former avec au maximum 1 individu par $\mathrm{g}$ de racine. Dans quelques rares cas, les femelles sont susceptibles de pondre un grand nombre d'œufs (320), mais, on peut considérer que, globalement, « Hiver du Prieuré » n'est pas un hôte de $M$. naasi. Les indices de galles pour cette avoine sont toujours inférieurs à ceux trouvés sur l'orge, que le potentiel infectieux soit faible ou fort.

Le nombre de femelles formées par g (tabl. 2) est significativement plus grand $(F=9,15)$ pour chacune des 5 variétés d'orge étudiées quand le potentiel infec-

\section{TABLEAU 2}

Influence des facteurs potentiels infectieux et variété d'orge et d'avoine d'hiver sur la formation des femelles, ceufs et galles de Meloidogyne naasi et sur le poids des racines de chaque plante.

Influence of degree of infection and winter variety of barley and oat on the production of females, eggs and galls of Meloidogyne naasi and on the root weight of each plant.

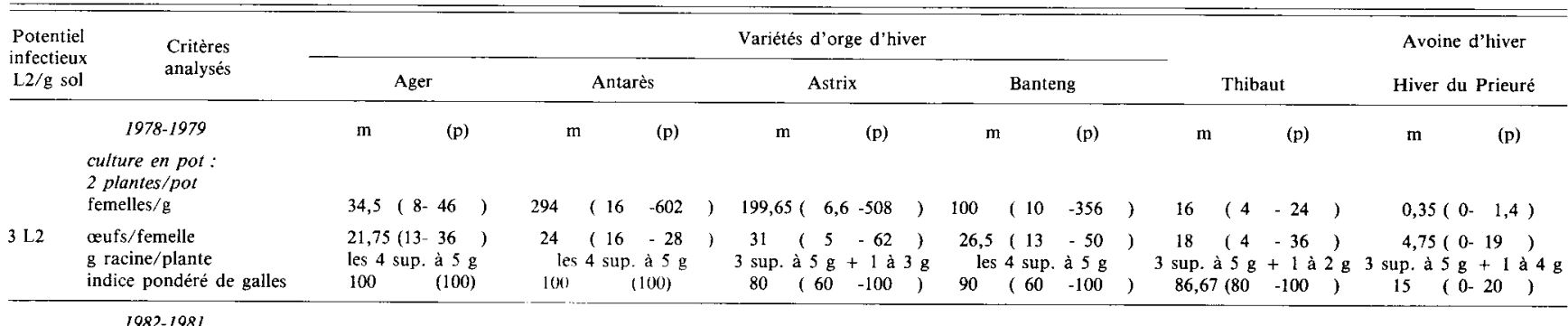

$1982-1981$

culture en pot :

$I$ plante/pot

femelles/g

cufs/femelle

g racine/plante

indice pondéré de galles

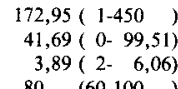

$3,89(2-6,06)$
80 $(60-100)$

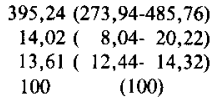

13,61
100

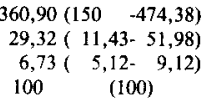

(100)

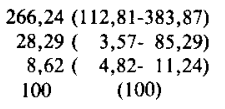

100

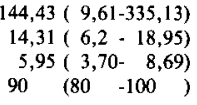

$0,36(0-1,4)$ $80 \quad(0-320)$ $5,92(4-8,27)$

$\mathrm{m}=$ moyenne sur 4 répétition

$\mathrm{p}=$ valeurs extrêmes : minimale et maximale

tieux est fort (18 L2). Cependant, un nombre croissant de femelles formées par g de racine est observé sur ces variétés d'orge classées comme suit : «Thibaut » «Ager », " Banteng », "Astrix » et « Antarès », quels que soient le potentiel infectieux et l'année d'expérience. Les orges peuvent être réparties en 2 groupes : «Antarès » et «Astrix » donnent significativement plus de femelles par $\mathrm{g}$ de racine que "Banteng ", «Ager» et « Thibaut» $(F=8,7)$. Quels que soient le potentiel infectieux ou la variété, les nombres d'œufs formés par femelle et les indices de galles ne sont pas significativement différents d'une plante à une autre.

\section{b) Les blés}

Quelles que soient les conditions d'expérience, au champ ou en pot, à faible ou fort taux d'infestation, la variété "Top » donne significativement plus de femelles par $g$ de racine $(F=8,7)$ que « Hardi » et « Capitole » (tabl. 3). L'emploi d'un fort potentiel infectieux (20 L2) augmente significativement l'indice pondéré de galles de chaque variété alors qu'il ne modifie pas le nombre de femelles formées. Les poids de racines des plantes cultivées au champ sont toujours significativement inférieurs à ceux des plantes semées en pot $(F=73,1)$.

\section{Qualité d'hôte d'A. ventricosa 11 (tabl. 4)}

$A$. ventricosa 11 , résistant à $H$. avenae, ne l'est pas vis-à-vis de $M$. naasi. Le fort potentiel infectieux de 23 L2 de l'essai 1981 est responsable de la formation d'un grand nombre de femelles par $g$ de racine, 114 en moyenne, et d'un indice pondéré de galles important. A faible potentiel infectieux ( $3 \mathrm{~L} 2)$ les résultats diffèrent nettement de ceux enregistrés pour $23 \mathrm{~L} 2$ et sont moins bons en ce qui concerne l'indice pondéré de galles, le nombre de femelles formées et le nombre d'œufs par femelle. Par contre, la date d'extraction n'a pas d'influence sur l'évaluation de la multiplication de $M$. naasi dans le cas de faible infestation (3 L2).

\section{B. Tests réalisés en boîtes de Petri}

Les avoines de type hiver ou printemps, « Hiver du Prieuré » et "Nelson », présentent pour toutes les conditions d'utilisation du test, un très grand nombre 
TABLEAU 3

Influence des facteurs potentiel infectieux et variétés de blé d'hiver sur la formation des femelles, oufs et galles de Meloidogyne naasi et sur le poids des racines de chaque plante.

Influence of degree of infection and winter wheat variety on the production of females, eggs and galls of Meloidogyne naasi and on the root weight of each plant.

\begin{tabular}{|c|c|c|c|c|}
\hline \multirow{3}{*}{$\begin{array}{l}\text { Potentiel } \\
\text { infectieux } \\
\mathrm{L} 2 / \mathrm{g} \text { sol }\end{array}$} & \multirow{3}{*}{ Critères analysés } & \multicolumn{3}{|c|}{ Variétés de blés d'hiver } \\
\hline & & Capitole & Hardi & Top \\
\hline & & (p) & (p) & (p) \\
\hline $20 \mathrm{~L} 2$ & $\begin{array}{l}\text { culture en pot : } 2 \text { plantes/pot } \\
\text { femelles/g } \\
\text { œufs/femelle } \\
\text { g racine/plante } \\
\text { indice pondéré de galles }\end{array}$ & $\begin{array}{r}63,98(56-78) \\
25,57(15,17-31,48) \\
5,54(4,25-6,50) \\
50 \quad(40-60)\end{array}$ & $\begin{array}{ll}92 & (66-130) \\
38,65 & (26,80-61,09) \\
5,40 & (3,77-6,78) \\
65 & (60-70)\end{array}$ & $\begin{array}{r}144(44-190) \\
18,06(11,5-26,31) \\
6,02(3,51-7,36) \\
72,50(50-80)\end{array}$ \\
\hline $7 \mathrm{~L} 2$ & $\begin{array}{l}\qquad 1980-1981 \\
\text { culture au champ : } \\
\text { femelles/g } \\
\text { œufs/femelle } \\
\text { g racine/plante } \\
\text { indice pondéré de galles }\end{array}$ & $\begin{array}{r}68,08(21,60-141,64) \\
46,43(19-88,10) \\
1,62(1-2,62) \\
37,75(20-60)\end{array}$ & $\begin{array}{r}76,96(3,69-162,36) \\
32,68(10,50-56,11) \\
1,14(0,55-1,81) \\
39,46(31,11-56,36)\end{array}$ & $\begin{array}{r}128,56(68,96-218,50) \\
32,70(20,47-51,67) \\
2,60(2,09-3,27) \\
54,04(30-73,33)\end{array}$ \\
\hline
\end{tabular}

$\mathrm{m}=$ moyenne sur 4 répétitions

$\mathrm{p}=$ valeurs extrêmes : minimale et maximale

TABLEAU 4

Influence du facteur expérience sur la formation des femelles, oufs et galles de Meloidogyne naasi et sur le poids de racine de chaque plante d'Aegilops ventricosa 11 .

Influence of the experimental factor on the production of females, eggs and galls of Meloidogyne naasi and on the root weight of each tested plant of Aegilops ventricosa 11.

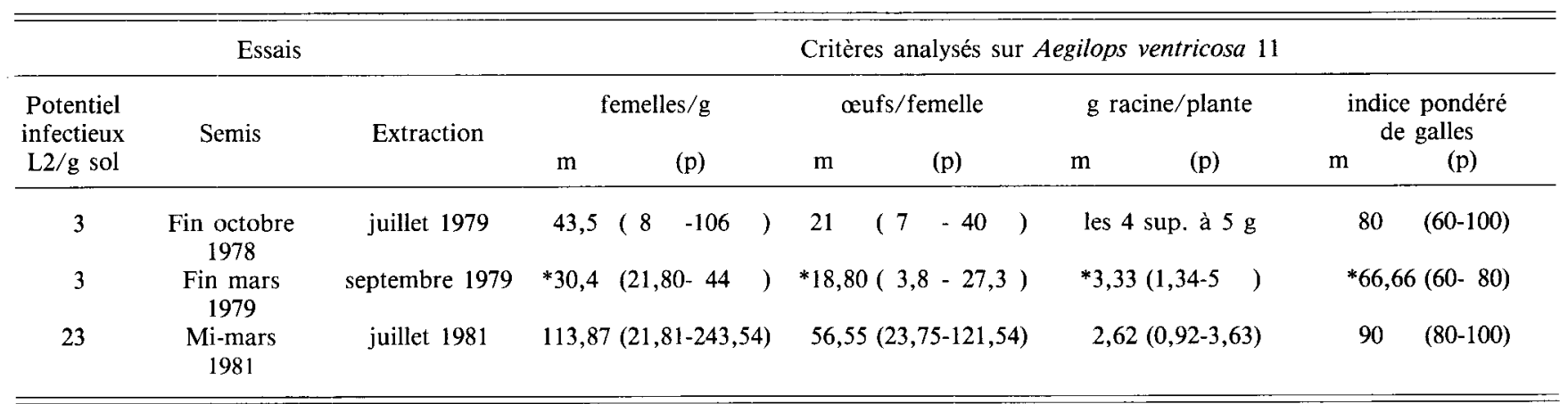

$\mathrm{m}=$ moyenne sur 4 répétitions, * 3 répétitions

$\mathrm{p}=$ valeurs extrêmes : minimale et maximale

de plantes sans femelles. Quelques-unes se forment cependant sur certaines plantes et sont capables de se reproduire mais restent toujours en nombre restreint. Les galles sont en nombre plus grand que les femelles (tabl. 5).

$A$. ventricosa 11 permet au maximum la formation d'une femelle par plante, et uniquement sur une très faible proportion des caryopses étudiés. Nous constatons que des galles se forment, quels que soient le potentiel infectieux et la température, leur nombre est en moyenne voisin ou supérieur à 2 (tabl. 5).

En ce qui concerne les espèces qui multiplient $M$. naasi, blés et orges de type hiver ou printemps, nous soulignerons plusieurs points qui ressortent de l'analyse de la formation des femelles et des œufs (tabl. 5) :

- seules les plantes soumises à un potentiel infectieux de 3 fois $16 \mathrm{~L} 2$ et entreposées à $23{ }^{\circ} \mathrm{C}$ présentent toutes au moins une femelle formée ;
- l'injection à la seringue d'environ 50 larves L2 par boîte de Petri stockées à 23 ou $20^{\circ} \mathrm{C}$, donne les meilleures moyennes de femelles formées pour 10 plantes d'une même variété, à l'exception de «Cornel » et d'《 Astrix », à $20^{\circ} \mathrm{C}$;

- parmi les tests utilisant le dépôt individuel des larves et pour une même température d'étude, ce sont les plus forts potentiels infectieux $(3 \times 16 \mathrm{~L} 2)$ qui donnent les plus grandes moyennes de femelles formées par variété, à l'exception de «Cérès » à $23^{\circ} \mathrm{C}$ et de «Cornel» à $20{ }^{\circ} \mathrm{C}$ :

- à potentiels infectieux identiques, c'est la température de $23{ }^{\circ} \mathrm{C}$ qui induit les meilleures moyennes de femelles formées par variété ;

- dans 26 des 43 tests réalisés pour les variétés de blé ou d'orge, chaque femelle donne une masse d'œufs, ce qui se traduit par une égalité des moyennes de masses d'œufs et de femelles formées pour 10 plantes d'une même variété. Dans les 17 autres cas, 
TABLEAU 5

Influence des facteurs température et potentiel infectieux sur la formation des femelles, des galles et œufs de Meloidogyne naasi sur diverses céréales cultivées en boites de Petri.

Influence of temperature and degree of infection on the production of females, galls and eggs of Meloidogyne naasi, on different cereals grown in Petri dishes.

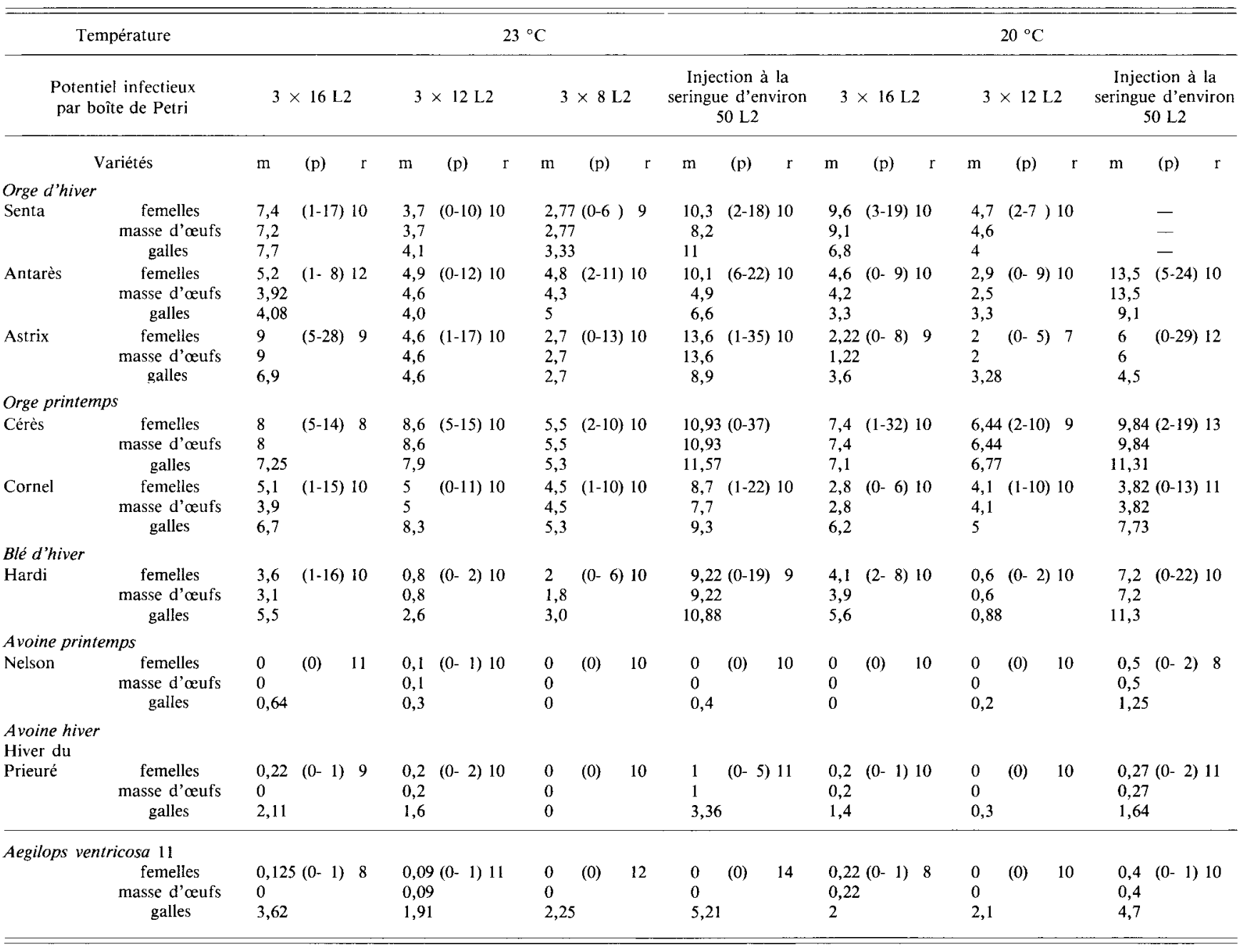

$\mathrm{m}=$ moyenne par boîte de Petri $-\mathrm{p}=$ valeurs extrêmes : minimale ou maximale trouvées par boîte de Petri $-\mathrm{r}=$ nombre de répétitions $(=$ nombre de boîtes de Petri)

ces moyennes sont très proches l'une de l'autre et montrent que seul un très petit nombre de femelles ne pond pas.

L'analyse de la moyenne des femelles formées par galle, pour les 41 tests réalisés sur orge ou blé (tabl. 5), montre qu'elle est égale ou très légèrement inférieure à 1 dans 27 tests. Elle dépasse 1 sans jamais atteindre 2 pour les 14 autres tests.

\section{DISCUSSION - CONCLUSIONS}

L'analyse critique des résultats obtenus dans les différents tests réalisés est nécessaire pour pouvoir préconiser un mode d'emploi des diverses méthodes de jugement de la résistance ou du caractère multiplicateur des céréales vis-à-vis de $M$. naasi. Ce travail doit apporter des renseignements que nous ne pouvons pas trouver dans les publications étrangères. Chaque auteur a en effet utilisé une technique particulière dans le but unique de réaliser les travaux qui l'intéressaient.
La comparaison des taux de multiplication obtenus chez différentes espèces, blé ou orge de type hiver ou printemps, ne sera pas faite dans ce travail. Elle fera l'objet d'une autre publication et sera réalisée sur un plus grand nombre de variétés par espèce étudiée.

\section{A. Analyse des tests réalisés sur sol infesté}

L'obtention de quelques rares femelles et œufs sur les 2 variétés d'avoine testées, "Noire de Moyencourt » et « Hiver du Prieuré », ne remet pas en cause le caractère résistant d' $A$. sativa déjà signalé par différents auteurs européens (FrANKLIN, 1965 ; CAUBEL $e t$ al., 1971 ; GOORIS \& D'HERDE, 1977). Ces auteurs trouvent également dans leurs tests quelques cultivars susceptibles de donner des nombres restreints de femelles et d'œufs.

Différents points, concernant les femelles, oufs et galles de $M$. naasi, ressortent de l'analyse des résultats obtenus avec les espèces multipliant le nématode: orge, blé et $A$. ventricosa 11 .

- Les nombres de femelles ou de galles trouvés sur 
chaque variété d'orge de printemps en juillet sont significativement plus importants que ceux notés en septembre. Le bon développement du nématode dans toutes les expériences réalisées sur orge de printemps se traduit par des nombres d'œufs formés et comptés par femelle statistiquement identiques dans tous les cas. Le lavage des plantes en septembre doit donc éliminer un plus grand nombre de galles, et donc de femelles, avant notation. A cette date, les plantes ont dépassé d'un mois la moisson, réalisée fin juillet ou début août dans nos régions, et ont un système racinaire plus fragile.

- L'augmentation du potentiel infectieux initial induit, pour chaque variété d'orge de printemps analysée en juillet, une augmentation de l'indice de galles et du nombre de femelles formées par $\mathrm{g}$ de racine. Chez les orges d'hiver, le taux d'infestation n'a une influence significative que sur la formation des femelles, puisque les indices de galles sont déjà proches de leur maximum (100) pour un faible nombre de larves L2 infestantes. L'échelle arbitrairement fixée dans l'établissement des indices de galles est sans doute responsable de l'apparition rapide de ce maximum.

Chez le blé d'hiver, l'augmentation des indices de galles avec le potentiel infectieux ne s'accompagne pas d'une formation de femelles plus élevée. Le caractère multiplicateur des blés vis-à-vis de $M$. naasi semble donc très limité lorsqu'il est comparé à celui des orges.

- Les tests restent très répétitifs malgré les changements de conditions de leur réalisation. Pour des variétés d'une même espèce de céréale, cultivées en pot ou en plein champ, le classement suivant un ordre croissant de femelles par $\mathrm{g}$ de racine reste plusieurs années consécutives le même, que le potentiel infectieux soit faible ou fort. Le poids racinaire des plantes cultivées au champ, toujours significativement inférieur ou différent d'une année à une autre, ne joue pas également sur ce facteur classement.

\section{B. Analyse des tests réalisés en boîtes de Petri}

Pour être transposable dans la pratique, le test en boîtes de Petri doit donner des résultats comparables à ceux obtenus sur sol infesté.

Pour toutes les conditions d'utilisation étudiées, les avoines peuvent être prises comme témoins de résistance, de la même façon que pour les tests réalisés sur sol infesté. Seules quelques femelles peuvent se développer et pondre sur un nombre restreint de caryopses.

Quels que soient le potentiel infectieux et la température, les orges et blés multiplicateurs de $M$. naasi dans les tests faits sur sol permettent également la formation des femelles dans les boîtes de Petri, à l'exception cependant de quelques répétitions. Seules les boîtes contenant le plus fort inoculum éprouvé $(3 \times 16 \mathrm{~L} 2)$ et stockées à $23{ }^{\circ} \mathrm{C}$ permettent la détection systématique de femelles sur chaque plante des variétés testées. L'injection à la seringue d'une cinquantaine de larves L2 par répétition, avec stockage des boîtes à $23^{\circ} \mathrm{C}$, permet la formation d'un plus grand nombre de femelles en moyenne par variété. Cette technique, réa- lisée aisément, peut permettre de juger rapidement et globalement, sur 10 répétitions, le comportement d'une variété vis-à-vis de $M$. naasi.

Les résultats trouvés pour $A$. ventricosa 11 sont assez surprenants, puisqu'il est classé résistant au même titre que les avoines. Il ne permet pas la formation des femelles du parasite : résultats en contradiction avec ceux enregistrés dans les tests réalisés sur sol infesté. La bonne formation des galles, en boîtes de Petri, sur cette espèce, prouve que les nématodes ont bien pénétré. La physiologie perturbée de cette plante cultivée en conditions contrôlées doit sans doute être responsable du mauvais développement du nématode jusqu'à l'état de femelles adultes.

\section{Propositions de modes d'utilisation des tests}

Les tests réalisés sur sol infesté sont les seuls qui puissent permettre l'établissement des taux de multiplication de $M$. naasi sur diverses céréales, correspondant à ceux enregistrés dans nos conditions de culture. Les résultats sont cependant longs à obtenir : 4 à 9 mois après le semis des plantes, suivant que les variétés testées sont de type printemps ou hiver. Pour réaliser un bon test, il est intéressant de signaler certains renseignements :

1) Quatre répétitions par variété testée sont nécessaires mais suffisantes.

2) Si nous voulons nous assurer de la bonne réalisation du test, il est recommandé d'introduire des témoins :

- un de résistance pris parmi les avoines : « Noire de Moyencourt » ou «Hiver du Prieuré » par exemple,

- deux de multiplication, choisis pour leurs niveaux différents de multiplication de $M$. naasi: "Georgie » et «Cérès » chez les orges de printemps par exemple.

3) Seuls les nombres de femelles et d'œufs formés donnent une idée exacte de la multiplication de $M$. naasi sur céréale à paille.

4) Les notations des tests doivent se faire impérativement en juillet, quelle que soit la variété étudiée.

5) Il est préférable de travailler à fort potentiel infectieux, avoisinant 18 à $23 \mathrm{~L} 2$, pour obtenir avec plus de facilité une distinction entre les variétés qui multiplient peu, moyennement ou fortement le nématode.

La recherche de variétés résistantes ou de géniteurs de résistance, l'étude de l'hérédité de la résistance, peuvent être réalisées soit sur sol infesté, soit en boîtes de Petri. Cependant les tests en boîtes, plus faciles à analyser au bout d'un court laps de temps (1,5 mois), semblent mieux adaptés à l'analyse d'un grand nombre de plantes nécessaire à la réalisation de telles études.

La technique préconisant l'inoculation des larves infestantes L2 à la seringue, avec stockage des boîtes à $23{ }^{\circ} \mathrm{C}$, peut être employée dans le cadre de la recherche de géniteurs de résistance. Par contre, si nous voulons connaître le caractère multiplicateur ou résistant de chaque plante testée dans le cadre d'une étude de l'hérédité de la résistance, il faut impérativement 
choisir un inoculum de $3 \times 16 \mathrm{~L} 2$ et un stockage des boîtes à $23{ }^{\circ} \mathrm{C}$.

Nous ne devons pas oublier également que le travail réalisé sur espèce sauvage, telle que Aegilops ventricosa Tausch. ne peut être fait actuellement que sur sol contaminé, si nous voulons obtenir des résultats fiables.

Reçu le 16 janvier 1984. Accepté le 20 juin 1984.

\section{RÉFÉRENCES BIBLIOGRAPHIQUES}

Allen M. W., Hart W. H., Baghott K. V., 1970. Crop rotation controls barley rootknot nematode at Tulelake. Calif. Agric., 24, 4-5.

Caubel G., Lemaire J. M., Rivoal R., 1971. Observations préliminaires sur l'attaque de diverses céréales par Meloidogyne naasi en présence d'Heterodera avenae et d'Ophiobolus graminis. Conférence sur les Nématodes du genre Meloidogyne. O.E.P.P., Antibes, 27-29 octobre 1971, $10 \mathrm{p}$.

Caubel G., Ritter M., Rivoal R., 1972. Observations relatives à des attaques du nématode Meloidogyne naasi Franklin sur céréales et graminées fourragères, dans l'Ouest de la France en 1970. C.R. Acad. Agric. Fr., 351-356.

Cook R., 1972. Reaction of some oat cultivars to Meloidogyne naasi. Plant Pathol., 21, 41-43.

Coolen W. A., D'Herde D. J., 1972. A method for the quantitative extraction of nematodes from plant tissue. Publ. of the Government. Res. Stn for Nematol. and Entomol., Merelbecke, Belgium, $77 \mathrm{p}$.

Dagnelie P., 1970. Théorie et méthodes statistiques. Applications agronomiques. II. Les méthodes de l'inférence statistique. Les Presses agronomiques Gembloux A, S, B, L.. Ed. J. Duculot S.A., Gembloux, 451 p.

Dosba F., Rivoal R., 1981. Les lignées d'addition Blé - Aegilops ventricosa Tausch.. II. Etude de leur comportement et de celui de leurs progéniteurs vis-à-vis d'Heterodera avenae Woll.. Agronomie, 1, 559-565.

Ediz S. A., Dickerson O. J., 1976. Life cycle, pathogenicity, histopathology and host range of race 5 of the barley root knot nematode. J. Nematol., 8, 228-232.

Franklin M. T., 1965. A root-knot nematode, Meloidogyne naasi sp., on field crops in England and Wales. Nematologica, 11, 79-86.

Gooris J., D'Herde C. J., 1977. Study on the biology of Meloidogyne naasi Franklin 1965. State Nematol. Entomol. Res. Stn. Merelbecke, Belgique, $165 \mathrm{p}$.
Kilpatrick R. A., Gilchrist L., Golden A. M., 1976. Root knot on wheat in Chile. Plant Dis. Rep., 60, 135.

Michell R. E., Malek R. B., Taylor D. P., Edwards S. D. J., 1973. Races of the barley root-knot nematode, Meloidogyne naasi. I. Characterization by host preference. J. Nematol., 5, 41-44.

Ogunfowora A. O., 1977. Reaction of barley to cereal root-knot nematode, Meloidogyne naasi. Plant Pathol., 26, 161-166.

Ogunfowora A. O., Evans A. A. F., 1977. Factors affecting the hatch of eggs of Meloidogyne naasi an example of diapause in a second stage larva. Nematologica, 23, 137-146.

Person F., Doussinault G., 1978. Influence de la température et des caractères des races d'Heterodera avenae Woll. sur la validité d'un test en conditions contrôlées utilisable en sélection des céréales. Ann. Amélior. Plant., 28, 513-527.

Person-Dedryver F., 1983. Meloidogyne naasi : nématode à galle des céréales. Journées d'études «Rotations céréalières intensives" O.N.I.C., 16-17 mai 1983, Paris, 9 p.

Radewald J. D., Pyeatt L., Shybuya F., Humphrey W., 1970. Meloidogyne naasi a parasite of turfgrass in Southern California. Plant Dis. Rep., 54, 940-942.

Rivoal R., Person F., Caubel G., Scotto la Massese C., 1978. Méthodes d'évaluation de la résistance des céréales au développement des nématodes: Ditylenchus dipsaci, Heterodera avenae et Pratylenchus spp.. Ann. Amélior. Plant., 28, 371-394.

Roberts P. A., Van Gundy S. D., Waines J. G., 1982. Reaction of wild and domesticated Triticum and Aegilops species to root knot nematodes (Meloidogyne). Nematologica, 28, 182-191.

Siddiqui I. A., Taylor D. P., 1970. Symptoms and varietal reaction of oats to the Illinois isolate of the barley root-knot nematode Meloidogyne naasi. Plant Dis. Rep., 54, 972-975.

York P. A., 1980. Relationship between cereal root-knot nematode Meloidogyne naasi and growth and grain yield of spring barley. Nematologica, 26, 220-229. 